\title{
Ramsey spectroscopy of bound atomic states with extreme-ultraviolet laser harmonics
}

\author{
I. Liontos, ${ }^{1}$ S. Cavalieri, ${ }^{1,2}$ C. Corsi, ${ }^{1}$ R. Eramo, ${ }^{1,3}$ S. Kaziannis, ${ }^{1}$ A. Pirri, ${ }^{1}$ E. Sali, ${ }^{2}$ and M. Bellini ${ }^{1,3, *}$ \\ ${ }^{1}$ LENS, Via Nello Carrara 1, 50019 Sesto Fiorentino, Firenze, Italy \\ ${ }^{2}$ Dipartimento di Fisica-Università di Firenze, and CNISM, Via G. Sansone 1, I-50019 Sesto Fiorentino, \\ Firenze, Italy \\ ${ }^{3}$ Istituto Nazionale di Ottica-CNR, Largo E. Fermi 6, I-50125 Firenze, Italy \\ *Corresponding author: bellini@ino.it
}

Received October 6, 2009; revised December 22, 2009; accepted February 3, 2010; posted February 4, 2010 (Doc. ID 118202); published March 11, 2010

\begin{abstract}
We report the experimental measurement of Ramsey interference fringes in the single-photon excitation to a high-lying bound state of atomic argon by pairs of phase-locked, time-delayed, extreme UV high-orderharmonic pulses at $87 \mathrm{~nm}$. High-visibility Ramsey fringes are observed for delays larger than $100 \mathrm{ps}$, thus demonstrating a potential resolving power $>10^{5}$ at this wavelength. (C) 2010 Optical Society of America OCIS codes: $190.4160,300.6560,320.7150$.
\end{abstract}

In recent years there has been increasing interest in the quest for high spectroscopic resolution in the vacuum and extreme UV (XUV) spectral regions. Synchrotron facilities can achieve a broad tunability in this wavelength range and in the $\mathrm{x}$ rays but, even when used in combination with the best available monochromators, they reach spectral resolutions only down to about $0.5-1 \mathrm{~cm}^{-1}$ [1], corresponding to a resolving power of about $10^{5}$. Together with their limited access, this has pushed research towards the development of novel techniques that exploit the tabletop characteristics of high-order harmonic $(\mathrm{HOH})$ sources. Unless generated with long nanosecond pulses (which, however, limit the extension toward short wavelengths), these, too, are inherently plagued by a broad bandwidth that has so far mainly limited their use to time-resolved studies. However, several recent experiments have shown that it is possible to gain high spectral resolution without losing the advantages of $\mathrm{HOH}$. Their general idea, based on that first introduced by Ramsey in 1950 [2], relies on extending the effective interrogation time of an atom or molecule by using pairs (or sequences) of collinear, phase-coherent, and time-delayed ultrashort pulses instead of a single broad-bandwidth one [3-7]. In principle, the spectral resolution $\Delta \nu$ achievable by these methods just depends on the maximum time delay $\tau$ between the exciting pulses and, like in Fourier transform spectroscopy (FTS), one gets $\Delta \nu \approx 1 / \tau$. If the delay is introduced by translating an optical delay line of a distance $L$ (like in one arm of a Michelson interferometer), the delay is $\tau=(2 L) / c$, where $c$ is the speed of light, and the resolving power achievable with a given mirror displacement is thus

$$
R=\frac{\nu}{\Delta \nu} \approx \frac{2 L}{\lambda}
$$

i.e., it simply corresponds to twice the number of wavelengths $(\lambda)$ scanned by the mirror movement. It is interesting to note that, in the case of XUV radiation with wavelengths in the $30-100 \mathrm{~nm}$ range, just a few millimeters of mirror displacement achieve the same resolving power of the best synchrotron monochromators.

Owing to the lack of suitable optics for creating a controlled pulse pair in the XUV, all the experiments aiming to extend similar techniques to $\mathrm{HOH}$ have been based on controlled manipulation (i.e., splitting and delaying) of the pump field and relied on the phase coherence of the generation process [8-10]. The first experimental demonstration of Ramseytype spectroscopy with $\mathrm{HOH}$ involved autoionizing states of krypton [11-13]. A similar approach has been recently used for the spectroscopy of a bound state at $125 \mathrm{~nm}$ in xenon and for a two-photon transition at $212 \mathrm{~nm}$ in krypton $[14,15]$. The feasibility of an XUV extension of the FTS technique was demonstrated with a similar scheme [16]. Finally, the use of trains of phase-locked pump pulses has shown the potential to produce a comb of narrow spectral modes in the harmonic spectrum [17-19].

Here we report the first (to our knowledge) use of the original two-pulse Ramsey scheme [11] to study high-lying bound atomic states, whose long-level lifetimes allow us to put to a stringent test the spectral resolution limits of the technique. A single-photon XUV transition at $87 \mathrm{~nm}$ in atomic argon is investigated. The upper $3 s^{2} 3 p^{5}\left({ }^{2} P_{1 / 2}\right) 3 d$ level lies close to the first ionization threshold of the atom, and excited electrons can be promoted to the continuum by absorption of a single further IR photon at the fundamental wavelength. Operating in a XUV-pump/IRprobe setup has involved an upgraded experimental scheme where a part of the fundamental IR light is used to photoionize the excited argon atoms (see Fig. 1). Although the latter is a rather standard technique in atomic spectroscopy, it is important to stress that it is used here for the first application of real (nonperturbative) high-order XUV harmonics to the spectroscopy of bound atomic states. The laser source is an amplified Ti:sapphire laser working at a repetition rate of $1 \mathrm{kHz}$ and providing $0.8 \mathrm{~mJ}, 30 \mathrm{fs}$ pulses at a wavelength of $800 \mathrm{~nm}$. The main part of the laser emission is sent to a semimonolithic Michelson interferometer that splits the incoming pulses and 


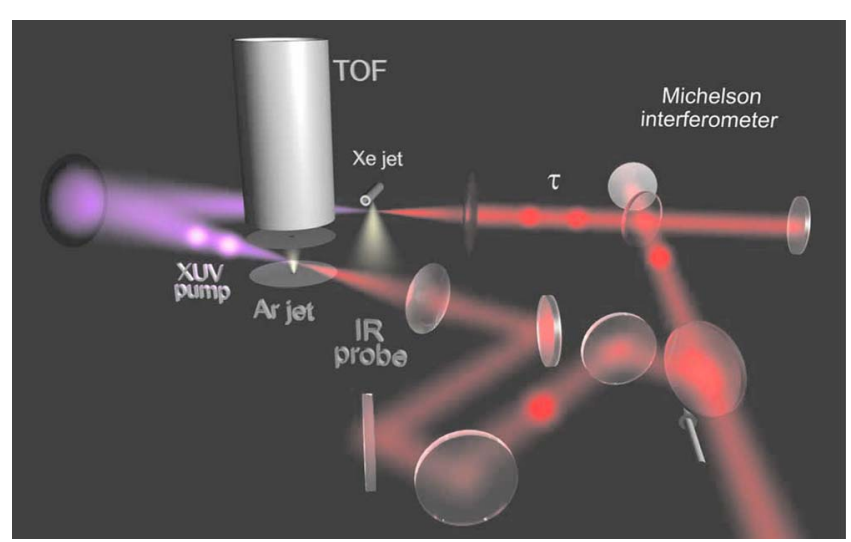

Fig. 1. (Color online) Schematic view of the experimental setup.

creates pairs of identical output pulses with a finely controllable phase delay. Accurate thermal and acoustic isolation provides an excellent stability of the relative optical paths in the two arms of the interferometer (better than $10-20 \mathrm{~nm} / \mathrm{min}$ ). This is a fundamental requirement for the realization of the experiment, since a relative jitter of the order of the harmonic wavelength would be sufficient to completely obscure the delicate interference signals. The pump-pulse pair is then focused by a $250 \mathrm{~mm}$ focal length lens into a vacuum chamber where a laserdrilled metal tube filled with xenon at a backing pressure of about 2 bar provides the continuous-flow gas source for harmonic generation. Different harmonic orders are dispersed by a normal incidence grating, and the ninth harmonic is refocused onto a second continuous gas jet of argon in an interaction chamber. The interaction between the argon atoms and the harmonic pulses takes place along the axis of a vertical time-of-flight (TOF) spectrometer, which collects the results of ionization with a small acceptance angle such that Doppler effects can be safely neglected in the analysis. Owing to the astigmatism of the optics, the spatial intensity distribution of the XUV beam in the interaction region is well focused (about $30 \mu \mathrm{m}$ ) in the vertical direction, while it has a very elongated horizontal profile (about $3 \mathrm{~mm}$ ). This particular configuration has the advantage of defining a precise vertical position for the ion/electron source region, thus preserving a high-energy resolution in the TOF while effectively canceling the effect of spectral dispersion introduced by the grating in the interaction region (for more details, see [12]). Probe IR pulses are obtained by intercepting a fraction of the laser emission with a wedged glass plate before entering the Michelson interferometer. After an appropriate delay line, the probe pulses with energies of the order of $20-30 \mu \mathrm{J}$ are focused onto the excited argon atoms in a counterpropagating direction with respect to the XUV beam. A delay of about $1 \mathrm{~ns}$ is imposed between the pair of exciting XUV pulses and the probe IR one. Experimental conditions are such that no ionization is observed from the IR pulses alone.

A typical ion mass spectrum after the excitation by the two phase-locked XUV harmonic pulses and the IR one is presented in Fig. 2(a). Clear contributions

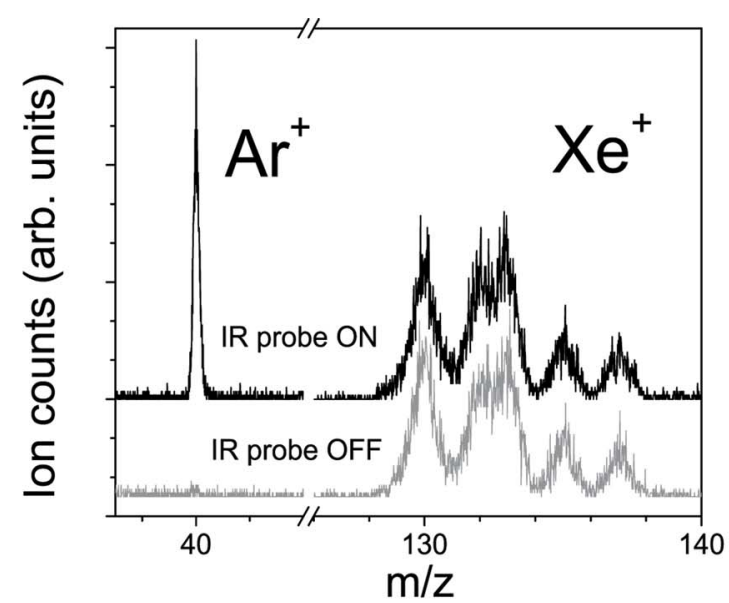

(a)
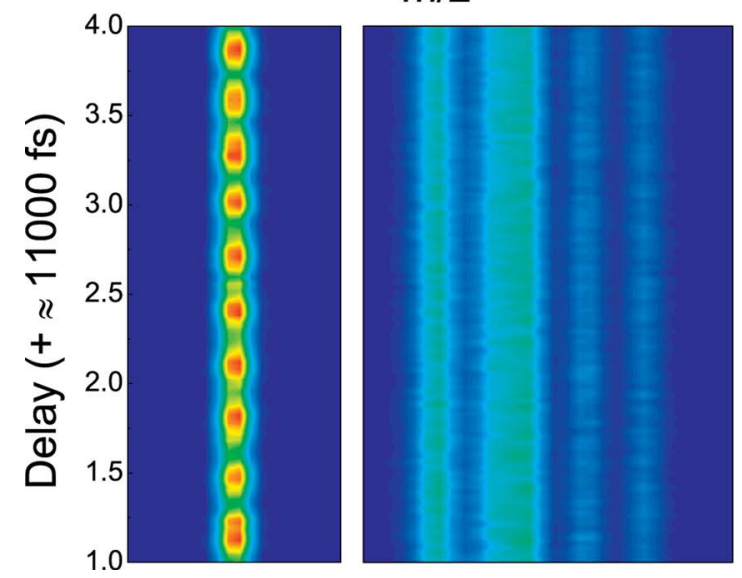

(b)

Fig. 2. (Color online) (a) Ion mass spectra with XUV pulses only (lower gray curve); with XUV pulses + delayed IR probe (upper black curve). (b) Ion mass spectra obtained by scanning the harmonic interpulse delay $\tau$.

from $\mathrm{Ar}^{+}$and $\mathrm{Xe}^{+}$ions are visible, with the latter due to residual atoms coming from the region of harmonic generation. $\mathrm{Xe}^{+}$ions are almost unaffected by the presence of the probe field, whereas the ionization of argon atoms requires the presence of the delayed IR probe pulse. When varying the delay $\tau$ between the harmonic pulses by means of a controlled sawtooth motion of a piezoelectric transducer in one interferometer arm, the number of $\mathrm{Ar}^{+}$ions exhibits evident oscillations of good visibility [see Fig. 2(b)]. On the contrary, no modulation appears in the excitation probability of Xe atoms, since ionization essentially proceeds via an unstructured continuum in this case. The level of xenon ionization thus just depends on the harmonic intensity and can be used as a reference for normalizing the measurements of $\mathrm{Ar}^{+}$yield. Ramsey fringes are the clear signature of a quantum interference between the ground and the excited level of argon atoms, which is induced by the first XUV pulse and probed by the second one. No modulation of the xenon signal was observed for delays larger than about $100 \mathrm{fs}$, showing that no residual optical interference between the delayed harmonic pulses is present and that only the effect of the atomic "memory" is responsible for the periodic modulation in the argon signal. We performed several fine delay scans at different positions of the moving interferometer arm. Starting from a delay close to 
$100 \mathrm{fs}$, we moved in coarse steps of approximately $20 \mathrm{ps}$, until the maximum delay of about $110 \mathrm{ps}$ allowed by our interferometer in the present configuration was reached (see Fig. 3). This corresponds to a total optical path difference of about $34 \mathrm{~mm}$ that, according to Eq. (1), results in a potential spectral resolution of $2 \times 10^{-4} \mathrm{~nm}$, or a resolving power of about $4 \times 10^{5}$ at $87 \mathrm{~nm}$.

In conclusion, we have demonstrated the potential of Ramsey spectroscopy based on $\mathrm{HOH}$ in reaching high spectral resolution in the XUV region with a relatively simple table-top setup. Differently from other approaches, our scheme does not require accurately phase-stabilized laser sources nor cumbersome experimental apparata and involves only a very limited scan of the moving arm of a Michelson interferometer to achieve an excellent resolving power. Such a scheme can be directly used to measure narrow transition linewidths in the XUV region (by measuring the fringe visibility decay) or to accurately determine line splittings (by observing the beat notes in the Ramsey interference fringes). In such cases, it is not necessary to accurately follow the fine evolution of the interference fringes, and just recording their envelope is sufficient to extract the desired quantities. Absolute frequencies of isolated transitions or congested spectra can be measured by applying Fourier-transformation techniques to a sufficiently large number of Ramsey fringes recorded (either continuously or for a proper set of subsamples) while accurately monitoring the interpulse delay.

Scaling the present setup to much larger delays in the range of tens of nanoseconds (corresponding to meters of arm displacements) will require substan-

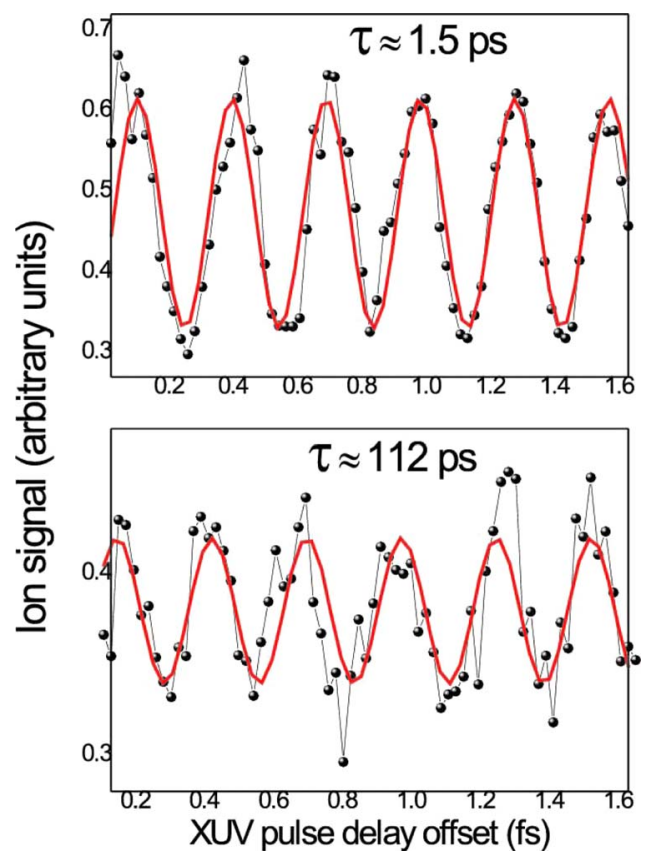

Fig. 3. (Color online) Ramsey fringes in the ionization signal of argon at coarse delays of 1.5 and $112 \mathrm{ps}$. The different fringe visibilities are mainly due to the difficulty in keeping the moving arm alignment for large delay scans. tial technical improvements in the stability and accurate monitoring of the moving arm position, together with a careful control over the beam propagation. If solutions in the line of those developed along the years for conventional FTS (including corner-cube reflectors to improve beam alignment, vacuum propagation to eliminate linear and nonlinear pulse distortions, optical references for accurate delay calibration, better harmonic generation and ion detection efficiency to increase the overall acquisition rate, etc.) are adopted here, a resolving power of $10^{8}$ in the XUV region is a realistic goal to be achieved.

Work partially supported by Ente Cassa di Risparmio di Firenze and by the European Commissions FP7 under grant agreement 228334. We acknowledge R. Ballerini, A. Hajeb, M. Giuntini, M. De Pas, and A. Montori for technical assistance.

\section{References}

1. P. A. Heimann, M. Koike, C. W. Hsu, D. Blank, X. M. Yang, A. G. Suits, Y. T. Lee, M. Evans, C. Y. Ng, C. Flaim, and H. A. Padmore, Rev. Sci. Instrum. 68, 1945 (1997)

2. N. F. Ramsey, Phys. Rev. 78, 695 (1950).

3. M. M. Salour, Rev. Mod. Phys. 50, 667 (1978).

4. J. T. Fourkas, W. L. Wilson, G. Wäckerle, A. E. Frost, and M. D. Fayer, J. Opt. Soc. Am. B 6, 1905 (1989).

5. M. Bellini, A. Bartoli, and T. W. Hänsch, Opt. Lett. 22, 540 (1997).

6. R. van Leeuwen, M. L. Bajema, and R. R. Jones, Phys. Rev. Lett. 82, 2852 (1999).

7. S. Cavalieri, M. Materazzi, R. Eramo, L. Fini, and A. Giugni, Opt. Commun. 182, 161 (2000).

8. R. Zerne, C. Altucci, M. Bellini, M. B. Gaarde, T. W. Hänsch, A. L'Huillier, C. Lyngå, and C.-G. Wahlström, Phys. Rev. Lett. 79, 1006 (1997).

9. M. Bellini, C. Lyngå, A. Tozzi, M. B. Gaarde, T. W. Hänsch, A. L'Huillier, and C.-G. Wahlström, Phys. Rev. Lett. 81, 297 (1998).

10. M. Bellini, S. Cavalieri, C. Corsi, and M. Materazzi, Opt. Lett. 26, 1010 (2001).

11. S. Cavalieri, R. Eramo, M. Materazzi, C. Corsi, and M. Bellini, Phys. Rev. Lett. 89, 133002 (2002).

12. A. Pirri, E. Sali, C. Corsi, M. Bellini, S. Cavalieri, and R. Eramo, Phys. Rev. A 78, 043410 (2008).

13. S. Cavalieri and R. Eramo, Phys. Rev. A 58, R4263 (1998).

14. S. Witte, R. T. Zinkstok, W. Ubachs, W. Hogervorst, and K. S. E. Eikema, Science 307, 400 (2005).

15. R. T. Zinkstok, S. Witte, W. Ubachs, W. Hogervorst, and K. S. Eikema, Phys. Rev. A 73, 061801 (2006).

16. M. Kovačev, S. V. Fomichev, E. Priori, Y. Mairesse, H. Merdji, P. Monchicourt, P. Breger, J. Norin, A. Persson, A. L'Huillier, C.-G. Wahlström, B. Carré, and P. Salières, Phys. Rev. Lett. 95, 223903 (2005).

17. R. J. Jones, K. D. Moll, M. J. Thorpe, and J. Ye, Phys. Rev. Lett. 94, 193201 (2005).

18. C. Gohle, T. Udem, M. Herrmann, J. Rauschenberger, R. Holzwarth, H. A. Schuessler, F. Krausz, and T. W. Hänsch, Nature 436, 234 (2005).

19. A. Ozawa, J. Rauschenberger, C. Gohle, M. Herrmann, D. R. Walker, V. Pervak, A. Fernandez, R. Graf, A. Apolonski, R. Holzwarth, F. Krausz, T. W. Hänsch, and T. Udem, Phys. Rev. Lett. 100, 253901 (2008). 